\title{
Fibonacci and Lucas numbers via the determinants of tridiagonal matrix
}

\author{
Taras Goy \\ Department of Mathematics and Informatics \\ Vasyl Stefanyk Precarpathian National University \\ 57 Shevchenko Str., 76018 Ivano-Frankivsk, Ukraine \\ e-mail: tarasgoyeyahoo.com
}

Received: 12 June 2016

Accepted: 31 January 2018

Abstract: Applying the apparatus of triangular matrices, we proved new recurrence formulas for the Fibonacci and Lucas numbers with even (odd) indices by tridiagonal determinants.

Keywords: Fibonacci numbers, Lucas numbers, Horadam sequence, Triangular matrix, Parapermanent of triangular matrix.

2010 Mathematics Subject Classification: 11B39, 11C20.

\section{Triangular matrix and parapermanents of triangular matrix}

The functions of triangular matrices are widely used in algebra, combinatorics, number theory and other branches of mathematics $[9,11,12]$.

Definition 1.1. [11]. A triangular number table

$$
A_{n}=\left(\begin{array}{cccc}
a_{11} & & & \\
a_{21} & a_{22} & & \\
\vdots & \vdots & \ddots & \\
a_{n 1} & a_{n 2} & \cdots & a_{n n}
\end{array}\right)
$$

is called a nth-order triangular matrix.

Note that a matrix (1) is not a triangular matrix in the usual sense of this term as it is not a square matrix. 
The product $a_{i j} a_{i, j+1} \cdots a_{i i}$ is denoted by $\left\{a_{i j}\right\}$ and is called a factorial product of the element $a_{i j}$

Definition 1.2. [11]. The parapermanent $\operatorname{pper}\left(A_{n}\right)$ of a triangular matrix (1) is the number

$$
\operatorname{pper}\left(A_{n}\right) \equiv\left[\begin{array}{cccc}
a_{11} & & & \\
a_{21} & a_{22} & & \\
\vdots & \vdots & \ddots & \\
a_{n 1} & a_{n 2} & \cdots & a_{n n}
\end{array}\right]_{n}=\sum_{r=1}^{n} \sum_{p_{1}+\ldots+p_{r}=n} \prod_{s=1}^{r}\left\{a_{p_{1}+\ldots+p_{s}, p_{1}+\ldots+p_{s-1}+1}\right\},
$$

where $p_{1}, p_{2}, \ldots, p_{r}$ are positive integers, $\left\{a_{i j}\right\}$ is the factorial product of the element $a_{i j}$.

Example 1.3. The parapermanent of a 4-th order matrix:

$$
\begin{gathered}
\operatorname{pper}\left(A_{4}\right)=\left[\begin{array}{llll}
a_{11} & & \\
a_{21} & a_{22} & \\
a_{31} & a_{32} & a_{33} & \\
a_{41} & a_{42} & a_{43} & a_{44}
\end{array}\right]= \\
=a_{41} a_{42} a_{43} a_{44}+a_{31} a_{32} a_{33} a_{44}+a_{21} a_{22} a_{43} a_{44}+a_{21} a_{22} a_{33} a_{44}+ \\
+a_{11} a_{42} a_{43} a_{44}+a_{11} a_{32} a_{33} a_{44}+a_{11} a_{22} a_{43} a_{44}+a_{11} a_{22} a_{33} a_{44} .
\end{gathered}
$$

To each element $a_{i j}$ of a matrix (1) we associate the triangular table of elements of matrix $A_{n}$ that has $a_{i j}$ in the bottom left corner. We call this table a corner of the matrix and denote it by $R_{i j}\left(A_{n}\right)$. Corner $R_{i j}\left(A_{n}\right)$ is a triangular matrix of order $(i-j+1)$, and it contains only elements $a_{r s}$ of matrix (1) whose indices satisfy the inequalities $j \leqslant s \leqslant r \leqslant i$.

Theorem 1.4. [11] (Decomposition of a parapermanent $\operatorname{pper}\left(A_{n}\right)$ by elements of the last row). The following formula are valid:

$$
\operatorname{pper}\left(A_{n}\right)=\sum_{s=1}^{n}\left\{a_{n s}\right\} \operatorname{pper}\left(R_{s-1,1}\left(A_{n}\right)\right),
$$

where $\operatorname{pper}\left(R_{0,1}\left(A_{n}\right)\right) \equiv 1$.

Example 1.5. Decomposition of a parapermanent $\operatorname{pper}\left(A_{4}\right)$ by elements of the last row:

$$
\operatorname{pper}\left(A_{4}\right)=a_{44} \operatorname{pper}\left(A_{3}\right)+a_{43} a_{44} \operatorname{pper}\left(A_{2}\right)+a_{42} a_{43} a_{44} \operatorname{pper}\left(A_{1}\right)+a_{41} a_{42} a_{43} a_{44} \operatorname{pper}\left(A_{0}\right),
$$

where $\operatorname{pper}\left(A_{1}\right)=a_{11}, \operatorname{pper} A_{0} \equiv 1$.

R. Zatorsky and I. Lishchynskyy [10, 13] established connection between the paradeterminats and the lower Hessenberg determinants by formula

$$
\operatorname{pper}\left(A_{n}\right)=\left|\begin{array}{cccccc}
\left\{a_{11}\right\} & 1 & 0 & \ldots & 0 & 0 \\
-\left\{a_{21}\right\} & \left\{a_{22}\right\} & 1 & \ldots & 0 & 0 \\
-\left\{a_{31}\right\} & -\left\{a_{32}\right\} & \left\{a_{33}\right\} & \ldots & 0 & 0 \\
\vdots & \vdots & \vdots & \ddots & \vdots & \vdots \\
-\left\{a_{n-1,1}\right\} & -\left\{a_{n-1,2}\right\} & -\left\{a_{n-1,3}\right\} & \ldots & \left\{a_{n-1, n-1}\right\} & 1 \\
-\left\{a_{n 1}\right\} & -\left\{a_{n 2}\right\} & -\left\{a_{n 3}\right\} & \ldots & -\left\{a_{n, n-1}\right\} & \left\{a_{n n}\right\}
\end{array}\right|,
$$

where $\left\{a_{i j}\right\}$ is factorial product of the element $a_{i j}$. 


\section{A connection between the Horadam numbers with even (odd) indices and parapermanents}

In [5] A. Horadam considered the sequence

$$
h_{1}=p, h_{2}=q, \quad h_{n}=h_{n-1}+h_{n-2}, n \geq 3,
$$

where $p$ and $q$ are arbitrary integer numbers. This sequence generalized the Fibonacci sequence:

$$
F_{1}=1, F_{2}=1, \quad F_{n}=F_{n-1}+F_{n-2}, n \geq 3,
$$

and the Lucas sequence:

$$
L_{1}=2, L_{2}=1, \quad L_{n}=L_{n-1}+L_{n-2}, n \geq 3 .
$$

Proposition 2.1. The following formula is valid:

$$
h_{2 n-1}=\left[\begin{array}{ccccccccc}
p & & & & & & & \\
\frac{h_{2}}{1} & 1 & & & & & & \\
0 & \frac{h_{4}}{h_{1}} & 1 & & & & & \\
0 & 0 & \frac{h_{6}}{h_{3}} & 1 & & & & \\
\vdots & \vdots & \vdots & \vdots & \ddots & & & \\
0 & 0 & 0 & 0 & \cdots & 1 & & \\
0 & 0 & 0 & 0 & \ldots & \frac{h_{2 n-4}}{h_{2 n-7}} & 1 & \\
0 & 0 & 0 & 0 & \cdots & 0 & \frac{h_{2 n-2}}{h_{2 n-5}} & 1
\end{array}\right] .
$$

Proof. Expanding the parapermanent (5) by elements of the last raw (see (3)), we have

$$
h_{2 n-1}=1 \cdot h_{2 n-3}+\frac{h_{2 n-2}}{h_{2 n-5}} \cdot h_{2 n-5}=h_{2 n-3}+h_{2 n-2} .
$$

Obtained equality holds by definition of the sequence $\left\{h_{n}\right\}_{n \geq 1}$.

Proposition 2.2. The following formula is valid:

$$
h_{2 n}=\left[\begin{array}{cccccccc}
q & & & & & & & \\
\frac{h_{3}}{1} & 1 & & & & & & \\
0 & \frac{h_{5}}{h_{2}} & 1 & & & & & \\
0 & 0 & \frac{h_{7}}{h_{4}} & 1 & & & & \\
\vdots & \vdots & \vdots & \vdots & \ddots & & & \\
0 & 0 & 0 & 0 & \cdots & 1 & & \\
0 & 0 & 0 & 0 & \cdots & \frac{h_{2 n-3}}{h_{2 n-6}} & 1 & \\
0 & 0 & 0 & 0 & \cdots & 0 & \frac{h_{2 n-1}}{h_{2 n-4}} & 1
\end{array}\right] .
$$

Proof. Using (3), we have

$$
h_{2 n}=1 \cdot h_{2 n-2}+\frac{h_{2 n-1}}{h_{2 n-4}} \cdot h_{2 n-4}=h_{2 n-2}+h_{2 n-1} .
$$




\section{Main results}

In this section we proved two recurrence formulas expressing the Horadam numbers $h_{n}$ by the determinant of tridiagonal matrix. As a consequence we received the corresponding formulas for the Fibonacci and Lucas numbers.

Proposition 3.1. The following formulas are valid:

$$
\begin{aligned}
& h_{2 n-1}=\frac{1}{h_{1} h_{3} \cdots h_{2 n-5}}\left|\begin{array}{ccccccccc}
p & 1 & 0 & 0 & 0 & \cdots & 0 & 0 & 0 \\
-h_{2} & 1 & 1 & 0 & 0 & \cdots & 0 & 0 & 0 \\
0 & -h_{4} & h_{1} & h_{1} & 0 & \cdots & 0 & 0 & 0 \\
0 & 0 & -h_{6} & h_{3} & h_{3} & \cdots & 0 & 0 & 0 \\
\vdots & \vdots & \vdots & \vdots & \vdots & \ddots & \vdots & \vdots & \vdots \\
0 & 0 & 0 & 0 & 0 & \cdots & -h_{2 n-4} & h_{2 n-7} & h_{2 n-7} \\
0 & 0 & 0 & 0 & 0 & \cdots & 0 & -h_{2 n-2} & h_{2 n-5}
\end{array}\right|, \\
& h_{2 n}=\frac{1}{h_{2} h_{4} \cdots h_{2 n-4}}\left|\begin{array}{ccccccccc}
q & 1 & 0 & 0 & 0 & \cdots & 0 & 0 & 0 \\
-h_{3} & 1 & 1 & 0 & 0 & \cdots & 0 & 0 & 0 \\
0 & -h_{5} & h_{2} & h_{2} & 0 & \cdots & 0 & 0 & 0 \\
0 & 0 & -h_{7} & h_{4} & h_{4} & \cdots & 0 & 0 & 0 \\
\vdots & \vdots & \vdots & \vdots & \vdots & \ddots & \vdots & \vdots & \vdots \\
0 & 0 & 0 & 0 & 0 & \cdots & -h_{2 n-3} & h_{2 n-6} & h_{2 n-6} \\
0 & 0 & 0 & 0 & 0 & \cdots & 0 & -h_{2 n-1} & h_{2 n-4}
\end{array}\right| .
\end{aligned}
$$

Proof. We prove the formula (7). From (5) using (4), we have

$$
h_{2 n-1}=\left|\begin{array}{ccccccccc}
p & 1 & 0 & 0 & 0 & \cdots & 0 & 0 & 0 \\
-\frac{h_{2}}{1} & 1 & 1 & 0 & 0 & \cdots & 0 & 0 & 0 \\
0 & -\frac{h_{4}}{h_{1}} & 1 & 1 & 0 & \cdots & 0 & 0 & 0 \\
0 & 0 & -\frac{h_{6}}{h_{3}} & 1 & 1 & \cdots & 0 & 0 & 0 \\
\vdots & \vdots & \vdots & \vdots & \vdots & \ddots & \vdots & \vdots & \vdots \\
0 & 0 & 0 & 0 & 0 & \cdots & -\frac{h_{2 n-4}}{h_{2 n-7}} & 1 & 1 \\
0 & 0 & 0 & 0 & 0 & \cdots & 0 & -\frac{h_{2 n-2}}{h_{2 n-5}} & 1
\end{array}\right| .
$$

After obvious simple transformations, we get (7).

Formula (8) can be proved similarly.

Example 3.2. Fibonacci numbers with odd indices:

$$
F_{2 n-1}=\frac{1}{F_{1} F_{3} \cdots F_{2 n-5}}\left|\begin{array}{ccccccccc}
1 & 1 & 0 & 0 & 0 & \cdots & 0 & 0 & 0 \\
-F_{2} & 1 & 1 & 0 & 0 & \cdots & 0 & 0 & 0 \\
0 & -F_{4} & F_{1} & F_{1} & 0 & \cdots & 0 & 0 & 0 \\
0 & 0 & -F_{6} & F_{3} & F_{3} & \cdots & 0 & 0 & 0 \\
\vdots & \vdots & \vdots & \vdots & \vdots & \ddots & \vdots & \vdots & \vdots \\
0 & 0 & 0 & 0 & 0 & \cdots & -F_{2 n-4} & F_{2 n-7} & F_{2 n-7} \\
0 & 0 & 0 & 0 & 0 & \cdots & 0 & -F_{2 n-2} & F_{2 n-5}
\end{array}\right|
$$


Example 3.3. The Fibonacci numbers with even indices:

$$
F_{2 n}=\frac{1}{F_{2} F_{4} \cdots F_{2 n-4}}\left|\begin{array}{ccccccccc}
1 & 1 & 0 & 0 & 0 & \cdots & 0 & 0 & 0 \\
-F_{3} & 1 & 1 & 0 & 0 & \cdots & 0 & 0 & 0 \\
0 & -F_{5} & F_{2} & F_{2} & 0 & \cdots & 0 & 0 & 0 \\
0 & 0 & -F_{7} & F_{4} & F_{4} & \cdots & 0 & 0 & 0 \\
\vdots & \vdots & \vdots & \vdots & \vdots & \ddots & \vdots & \vdots & \vdots \\
0 & 0 & 0 & 0 & 0 & \cdots & -F_{2 n-3} & F_{2 n-6} & F_{2 n-6} \\
0 & 0 & 0 & 0 & 0 & \cdots & 0 & -F_{2 n-1} & F_{2 n-4}
\end{array}\right| .
$$

Example 3.4. The Lucas numbers with odd indices:

$$
L_{2 n-1}=\frac{1}{L_{1} L_{3} \cdots L_{2 n-5}}\left|\begin{array}{ccccccccc}
2 & 1 & 0 & 0 & 0 & \cdots & 0 & 0 & 0 \\
-L_{2} & 1 & 1 & 0 & 0 & \cdots & 0 & 0 & 0 \\
0 & -L_{4} & L_{1} & L_{1} & 0 & \cdots & 0 & 0 & 0 \\
0 & 0 & -L_{6} & L_{3} & L_{3} & \cdots & 0 & 0 & 0 \\
\vdots & \vdots & \vdots & \vdots & \vdots & \ddots & \vdots & \vdots & \vdots \\
0 & 0 & 0 & 0 & 0 & \cdots & -L_{2 n-4} & L_{2 n-7} & L_{2 n-7} \\
0 & 0 & 0 & 0 & 0 & \cdots & 0 & -L_{2 n-2} & L_{2 n-5}
\end{array}\right| .
$$

Example 3.5. The Lucas numbers with even indices:

$$
L_{2 n}=\frac{1}{L_{2} L_{4} \cdots L_{2 n-4}}\left|\begin{array}{ccccccccc}
1 & 1 & 0 & 0 & 0 & \cdots & 0 & 0 & 0 \\
-L_{3} & 1 & 1 & 0 & 0 & \cdots & 0 & 0 & 0 \\
0 & -L_{5} & L_{2} & L_{2} & 0 & \cdots & 0 & 0 & 0 \\
0 & 0 & -L_{7} & L_{4} & L_{4} & \cdots & 0 & 0 & 0 \\
\vdots & \vdots & \vdots & \vdots & \vdots & \ddots & \vdots & \vdots & \vdots \\
0 & 0 & 0 & 0 & 0 & \cdots & -L_{2 n-3} & L_{L n-6} & L_{2 n-6} \\
0 & 0 & 0 & 0 & 0 & \cdots & 0 & -L_{2 n-1} & L_{2 n-4}
\end{array}\right| .
$$

Note, that determinants of matrices, elements of which are classical or generalized Fibonacci numbers, in particular, studied in $[1,2,3,4,6,7,8]$.

\section{Acknowledgements}

The author is grateful to professor Roman Zatorsky, Department of Mathematics and Informatics, Vasyl Stefanyk Precarpathian National University (Ukraine), for constant attention to this work and for useful discussions.

\section{References}

[1] Civciv, H. (2008) A note on the determinant of five-diagonal matrices with Fibonacci numbers, Int. J. Contemp. Math. Sciences, 3(9), 419-424. 
[2] İpek, A. (2011) On the determinants of pentadiagonal matrices with the classical Fibonacci, generalized Fibonacci and Lucas numbers, Eurasian Math. J., 2(2), 60-74.

[3] İpek, A., K. Ar1 (2014) On Hessenberg and pentadiagonal determinants related with Fibonacci and Fibonacci-like numbers, Appl. Math. Comput., 229, 433-439.

[4] Jaiswal, D. V. (1969) On determinants involving generalized Fibonacci numbers, Fibonacci Quart., 7(3), 319-330.

[5] Horadam, A.F. (1961) A generalized Fibonacci Sequence, Amer. Math. Monthly, 68, $455-459$.

[6] Koshy, T. (2001) Fibonacci and Lucas Numbers with Applications, New York: John Wiley \& Sons.

[7] Kwong, H. (2007) Two determinants with Fibonacci and Lucas entries, Appl. Math. Comput., 194(2), 568-571.

[8] Tangboonduangjit, A., T. Thanatipanonda (2015) Determinants containing powers of generalized Fibonacci numbers, http://arxiv.org/pdf/1512.07025.pdf.

[9] Zatorsky, R. (2015) Introduction to the theory of triangular matrices (tables). In: I. I. Kyrchei (eds.) Advances in Linear Algebra Research, New York: Nova Science Publishers, 185-238.

[10] Zatorsky, R. A. (2002) On paradeterminants and parapermanents of triangular matrices, Matematychni Studii, 17(1), 3-17 (in Ukranian).

[11] Zatorsky, R. A. (2007) Theory of paradeterminants and its applications, Algebra Discrete Math., 1, 108-137.

[12] Zatorsky, R., T. Goy (2016) Parapermanents of triangular matrices and some general theorems on number sequences, J. Integer Seq., 19(2), Article 16.2.2.

[13] Zatorsky, R. A., I. I. Lishchynskyy (2006) On connection between determinants and paradeterminants, Matematychni Studii, 25(1), 97-102 (in Ukranian). 